\title{
Analysis of Laminated Composite Plates by Meshless Method using FSDT
}

\author{
Mounesh V. Badiger and Dr.R.J. Fernandes
}

\begin{abstract}
Meshless method is a new type of numerical analysis that rectifies the drawbacks of mesh based methods like FEM (Finite Element Method). Meshless method is based on Element free Galerkin method and is developed for the laminated composite plates for flexural analysis. First order shear deformation method is employed in this work to the displacement shape functions. For the results, suitable boundary conditions are adopted. The accuracy of the solution obtained is established by comparing the results with the exact and FEM, solution which is available in literature.
\end{abstract}

Keywords--- Finite Element, Plate Theory, Meshless, AMD FSDT

\section{INTRODUCTION}

$\mathrm{P}$ LATE: It is a plane or flat surface and thickness is less as compared to length. Plate theories:

- Thin plate of small deflection.

- Thin plate of large deflection.

- Thick plates.

If the plates which are having a thickness less than $1 / 20^{\text {th }}$ of lateral side and deflection less than $1 / 5^{\text {th }}$ of the thickness it is called thick plate. In thick plates the thickness of plate will be large and they are used in the case of highly concentrated loads. Thin and thick plates having same concepts in the theories but the sizes are varying in thickness.

Finite Element method is a technique used for the solution of differential equations. FEM consists of generalization of classical variational i.e. Raleigh-Ritz Method and weightresidual i.e. Galerkin, Least-square. The problems which are defined will be complex and may have different type of boundary conditions in real world, so it is difficult to generate approximation using traditional variation methods.

In FEM the irregular boundaries are represented by the collections of finite elements this makes the solution to be simple. By using interpolation theory, the approximation function can be constructed and hence they are called as interpolation or Piecewise application of weighted-residual and variational methods.

In Classical laminate theory (CLT) it neglects shear deformation in the laminates along thickness like flat structures the CLT fails due to this shear deformation theory is recommended. The extension of Mindlin's i.e. First order

Mounesh V.Badiger, Mtech(CAADS), Student, Department of Civil Engg, SDMCET, Dharwad, India. E-mail:mouni.badiger406@gmail.com

Dr.R.J. Fernandes, Assistant Proffesor, Department of Civil Engg, SDMCET, Dharwad, India.E-mail:fernandes.regi@gmail.com DOI:10.9756/BIJMMI.8168 shear deformation theory (FSDT) is adopted for laminated anisotropic plates for transverse shear deformation.

The ML's (Meshless methods) is one of the numerical based analyses. These help in overcoming the disadvantages of the conventional approaches of Finite element method (FEM). When it comes to the study of mechanics and propagation of cracks, FEM has drawback were as Meshless techniques allow on substitute performance. The aerospace and other industry as a wide field of function with composite laminates as structural components. As compare to isotropic materials, the composite laminates are becoming progressively complicated in such cases these types of materials are studied using Meshless method.

\section{A. Classification of Meshfree Methods}

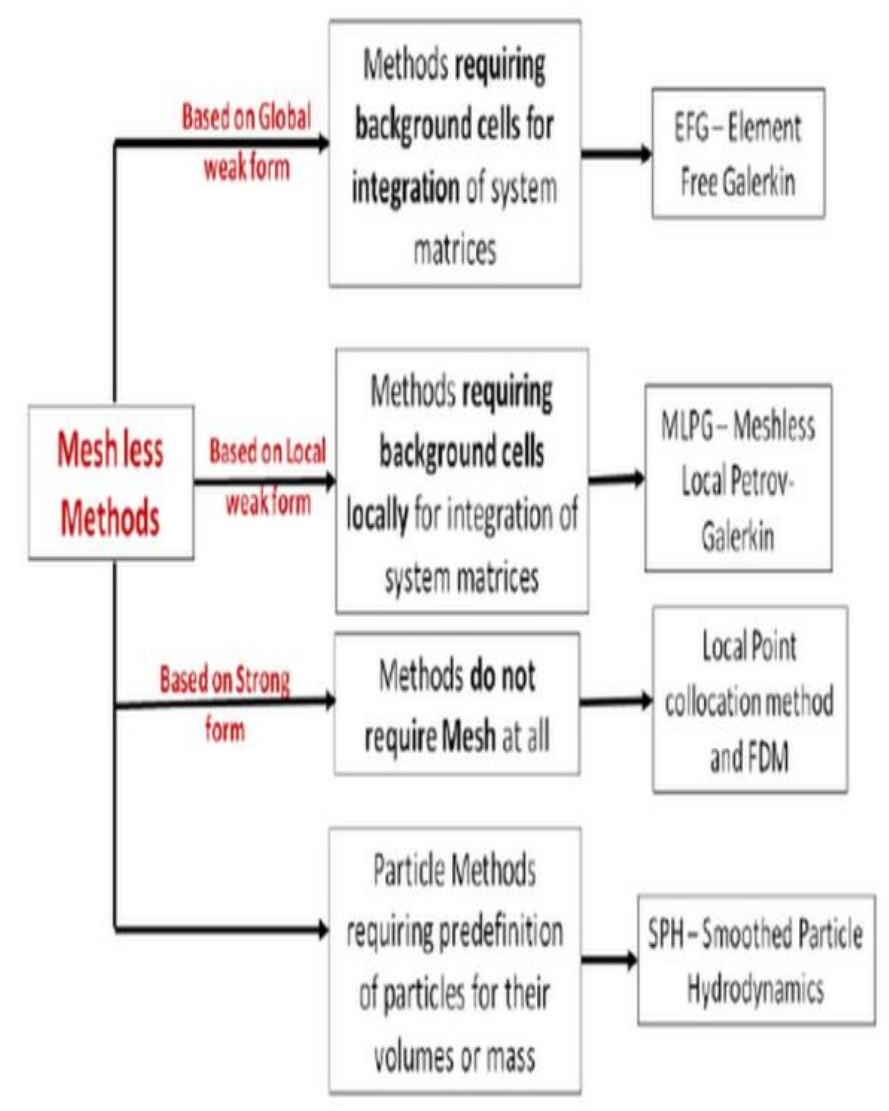

Figure 1: Classification of Meshfree Methods

\section{B. Objective}

Aim: The aim of the project is to analyse the laminated composite plates using Meshless method and to study the response of a plate subjected to static loading. 


\section{Scope of the Study}

The performance of the meshless method compared to FEM is done by applying the Meshless methods to the laminated composite plates under static loading :

- To develops the shape functions for the meshless methods.

D. Methodology
- To develops the algorithms found which are based on EFGM in relevant theories of structural.

- For the Plane stress and plate bending problems apply the Meshless methods in both laminates and isotropic plates.

- To check validating the results are compared with the literature and interpolation of results

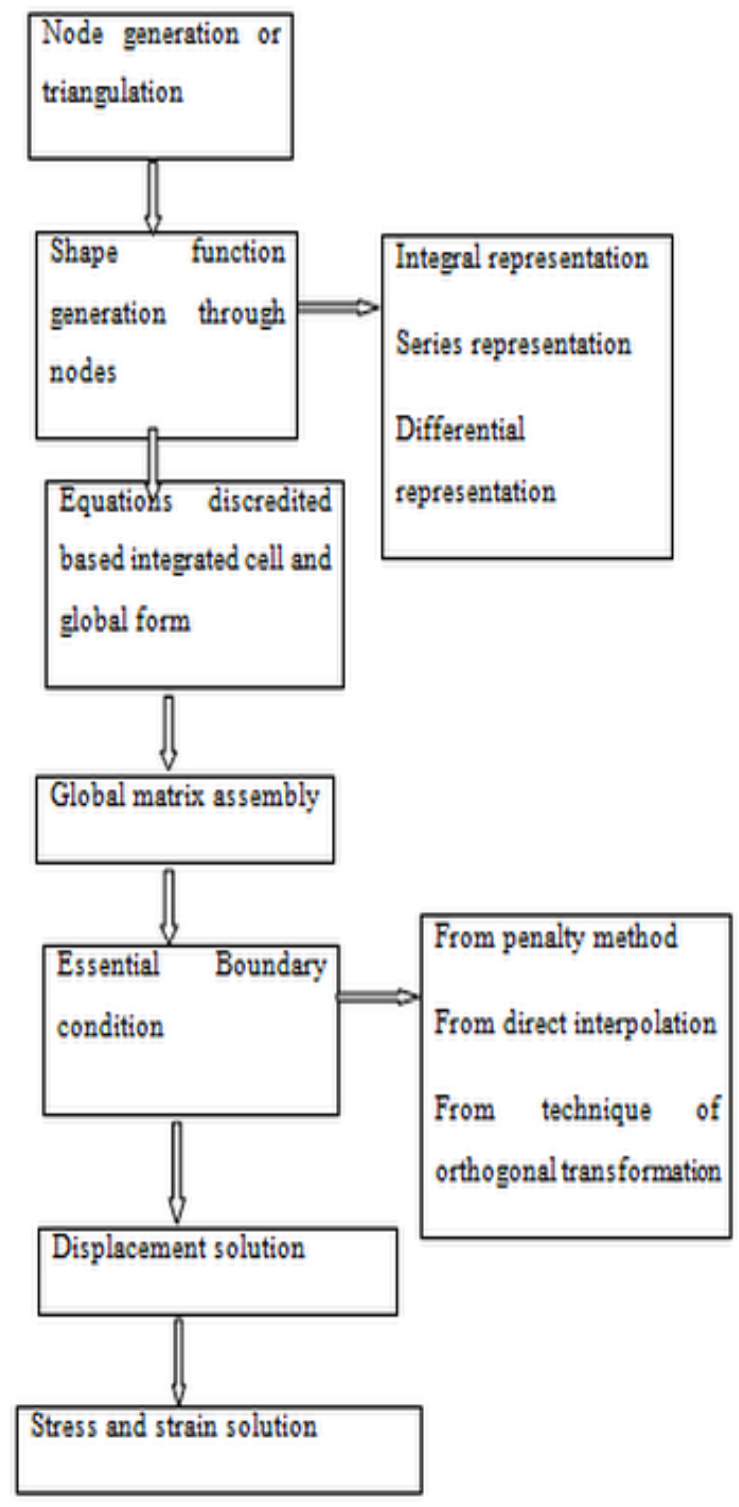

\section{E. Literature}

Wang (2002): In this paper, by using reproducing kernel particle method to calculate the parameters of laminated composite plates. In this approach the first order shear deformation (FSDT) is employed and displacement shape functions are obtained using RKPM method. Numerical problems are solved by various boundary conditions .
Figure 2: Methodology

Nayroles et al (1992): This paper is based on the week form and used Moving Least Square method and later was extended which is explained in next review .

J. N. REDDY (1989): Review was made based on the Finite Element models, continuum based theory, \& plate theory is used in the analysis of composite laminated plate upto $3^{\text {rd }}$ order, the shear deformation theories are studied. The study was made under various boundary conditions and laminated schemes. 


\section{Plate TheORETICAL Formulation}

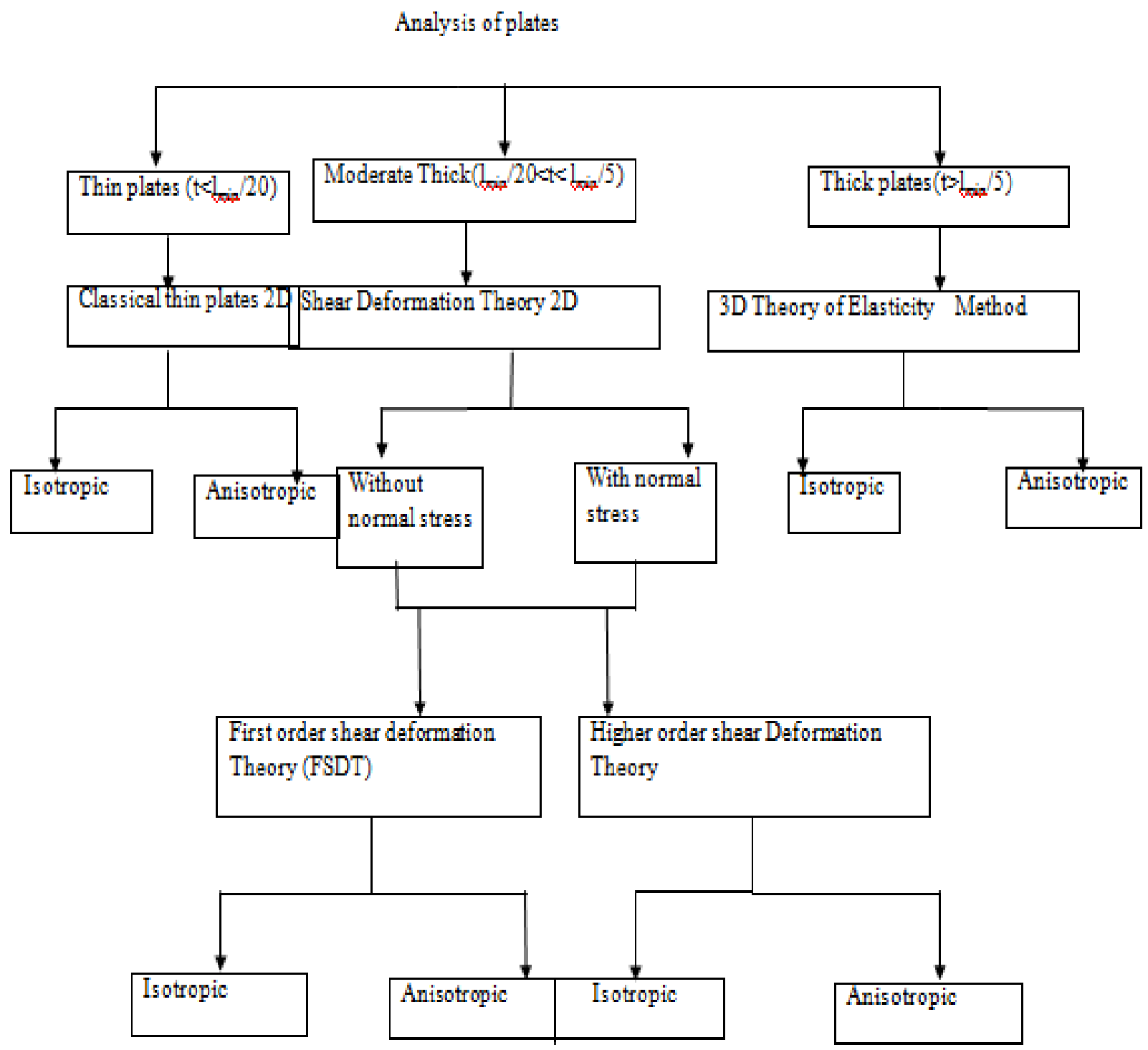

Figure 3: Flow Chart of Analysis of Plates

\section{A. Plate Theory}

1. In classical thin plate theory of analysis, it is assumed that plane sections normal to the undeformed midsurface remain plane and normal to the deformed mid surface after deformation analogous to Bernoulli-Euler theory of bending of beams.

2. As in the Timoshenko theory for analysis of deep beam the shear deformation theories taken as consideration of the transverse shear strain.

a. In the order Shear deformation theory, plane sections normal to the undeformed mid surface is assumed to be as remains plane but not normal to deformed mid surface after deformation the displacements taken as linear functions.

b. In HSDT plane sections normal to the undeformed mid-surface will not remain plane after deformation i.e. they undergo distortions as shown in the figure. This is because in higher order theories the displacement components are assumed to be in the form of power series like $\mathrm{u}=$ $\mathrm{A} 0+\mathrm{A} 1 \mathrm{z}+\mathrm{A} 2 \mathrm{z} 2+\ldots \ldots . .$.

3. In CTPT (Classical thin plate theory) as well as shear deformation theory the transverse displacements are assumed to be small and correspondingly the slopes of the tangents to the mid-surface are also assumed to be small. Consequently, the squares of the slopes may be neglected.

4. In situations where transverse displacement can't be taken to be small we have to include terms corresponding to the squares of the slopes in formulating the theories resulting in non-linear differential equation defining the problem. These theories are called large deformation theories. 


\section{B. Classical Thin Plate Theories}

The plates are moderately thick which are taken in this work. Hence the use of FSDT \& EFGM is used to obtain parameters.

In plane stress only two degree of freedom will be used i.e. in plane stresses \& displacements are considered. The matrix....

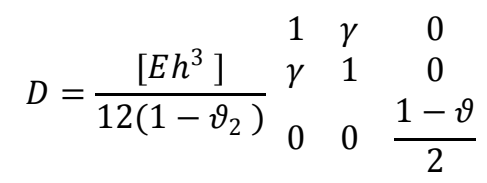

$\mathrm{E}=$ Youngs modulus

$$
\vartheta=\text { Poissons ratio }
$$

The equations which are given above holds good only for isotropic materials were as the relationship for orthotropic material is given below.

$$
Q_{i j}=\frac{1}{1-\vartheta_{12} \vartheta_{21}}\left[\begin{array}{ccc}
E_{1} & \vartheta_{21} E_{1} & 0 \\
\vartheta_{12} E_{2} & E_{2} & 0 \\
0 & 0 & G_{2}
\end{array}\right.
$$

The above relation holds good for only single laminate

For FSDT, the displacements of the laminated composite plate are

Expressed as:

$$
\begin{aligned}
& \mathrm{u}(\mathrm{x}, \mathrm{y}, \mathrm{z})=u_{0}(\mathrm{x}, \mathrm{y})+\mathrm{z} \theta_{x}(\mathrm{x}, \mathrm{y}) \\
& \mathrm{v}(\mathrm{x}, \mathrm{y}, \mathrm{z})=v_{0}(\mathrm{x}, \mathrm{y})+\mathrm{z} \theta_{y}(\mathrm{x}, \mathrm{y}) \\
& \mathrm{w}(\mathrm{x}, \mathrm{y}, \mathrm{z})=w_{0}(\mathrm{x}, \mathrm{y}) \\
& \mathrm{u}, \mathrm{v}, \mathrm{w}=\text { Displacement of a point in }(\mathrm{x}, \mathrm{y}, \mathrm{z}) \\
& \mathrm{u}_{0}, \mathrm{v}_{0}, \mathrm{w}_{0}=\text { Displacement on the mid plane } \\
& \theta \mathrm{x}, \theta \mathrm{y}=\text { rotation of the transverse and normal about } \mathrm{y} \text { and } \mathrm{x}
\end{aligned}
$$
axis

Using FSDT plate, displacement, bending shear strains could be obtained which are discussed in next chapter.

\section{Plate Theories}

Plates must be analyzed as a 3D object, such approach is reasonable in calculation. Some assumptions are made for plates and analyzed as 2D object.

There are 4 categories of plates:
1) Stiff plates
2) Thick plates
3) Moderate thick
4) Membranes

\section{Classical Laminate Theory}

Kirchhoff's theory which is applicable to thin plate laminates is called classical laminate theory. The shear and strain components are not taken into account in this theory. This is applicable only for thin plate because of absence of transverse shear. The variations of strain are assumes to be linear along thickness.
The expressions of strains are

$$
\begin{array}{r}
\epsilon_{x}=\frac{\partial \mathrm{u}}{\partial \mathrm{x}} \epsilon_{y}=\frac{\partial \mathrm{v}}{\partial \mathrm{y}} \gamma_{x y}=\frac{\partial \mathrm{u}}{\partial \mathrm{y}}+\frac{\partial \mathrm{v}}{\partial \mathrm{x}} \\
\mathrm{u}=\mathrm{u}_{0}-\mathrm{z} \partial \mathrm{w}_{0} / \partial \mathrm{x} \quad \mathrm{v}=\mathrm{v}_{0}-\mathrm{z} \partial \mathrm{w}_{0} / \partial \mathrm{y}
\end{array}
$$

The transformation between displacement and strains are shown in the equation below

$$
\left\{\begin{array}{c}
\epsilon_{x} \\
\epsilon_{y} \\
\gamma_{x y}
\end{array}\right\}=\begin{array}{ccc}
\frac{\partial}{\partial \mathrm{x}} & 0 & -\frac{\partial^{2}}{\partial \mathrm{x}^{2}} \\
0 & \frac{\partial}{\partial \mathrm{y}} & -\frac{\partial^{2}}{\partial \mathrm{y}^{2}} \\
\frac{\partial}{\partial \mathrm{y}} & \frac{\partial}{\partial \mathrm{x}} & -\frac{\partial^{2}}{\partial \mathrm{x} \partial \mathrm{y}}
\end{array}\left\{\begin{array}{l}
u_{0} \\
v_{0} \\
w_{0}
\end{array}\right\}
$$

\section{First Order Deformation Theory}

In FSDT plate theory the curvature at mid plane and bending shear strains changes and are defined as:

Strain displacement relation:

The $\mathrm{u}, \mathrm{v}, \mathrm{w}$ are the displacements of particle in the body $\mathrm{x}$, $\mathrm{y} \& \mathrm{z}$ displacements then the strain is given by:

$$
\epsilon_{x}=\frac{\partial \mathrm{u}}{\partial \mathrm{x}} ; \quad \epsilon_{y}=\frac{\partial \mathrm{v}}{\partial \mathrm{y}} ; \epsilon_{z}=\frac{\partial \mathrm{w}}{\partial \mathrm{z}} ; \gamma_{x y}=\frac{\partial \mathrm{u}}{\partial \mathrm{y}}+\frac{\partial \mathrm{v}}{\partial \mathrm{x}}
$$

If $\mathrm{u}_{0}, \mathrm{v}_{0}, \&_{\&} \mathrm{~W}_{0}$ are displacements of point $\mathrm{B}$ in $\mathrm{x}, \mathrm{y}, \mathrm{z}$ displacement let's consider general point "c" @ a Dist "z" from mid-plane before bending.

Therefore displacement of $\mathrm{c}$ in $\mathrm{x}$ displacement

$$
\mathrm{u}=\mathrm{u}_{0}-\mathrm{z} \beta=\mathrm{u}_{0}-\mathrm{z} \frac{\partial \mathrm{w}_{0}}{\partial \mathrm{x}}
$$

Therefore $\mathrm{u}=\mathrm{u}_{0}-\mathrm{Z}\left(\frac{\partial \mathrm{w}_{0}}{\partial \mathrm{x}}\right)$

Similarly in y displacement

wkt

$$
\mathrm{v}=\mathrm{v}_{0}-\mathrm{z} \frac{\partial \mathrm{w}_{0}}{\partial \mathrm{y}}
$$

$$
\begin{gathered}
\epsilon_{x}=\frac{\partial \mathrm{u}}{\partial \mathrm{x}}, \mathrm{u}=\mathrm{u}_{0}-\mathrm{z} \frac{\partial \mathrm{w}_{0}}{\partial \mathrm{x}} \\
\frac{\partial \mathrm{u}}{\partial \mathrm{x}}=\left(\frac{\partial \mathrm{u}}{\partial \mathrm{x}}\right)-\mathrm{z}\left(\frac{\partial^{2} w}{\partial \mathrm{x}^{2}}\right) \\
\epsilon_{x}=\left(\frac{\partial \mathrm{u}_{0}}{\partial \mathrm{x}}\right)-\mathrm{z}\left(\frac{\partial^{2} w_{0}}{\partial \mathrm{x}^{2}}\right) \\
\epsilon_{x}=\varepsilon_{\mathrm{x} 0}-\mathrm{z}\left(\mathrm{u}_{0}\right)
\end{gathered}
$$

Similarly $\epsilon_{y}=\left(\frac{\partial \mathrm{v}_{0}}{\partial \mathrm{y}}\right)-\mathrm{z}\left(\frac{\partial^{2} w_{0}}{\partial \mathrm{y}^{2}}\right)$

$$
\begin{gathered}
\epsilon_{y=} \varepsilon_{\mathrm{y} 0}-\mathrm{z}(\kappa \mathrm{y}) \\
\epsilon_{y=}\left(\frac{\partial^{2} w_{0}}{\partial \mathrm{y}^{2}}\right) \gamma_{x y}=\frac{\partial \mathrm{u}}{\partial \mathrm{y}}+\frac{\partial \mathrm{v}}{\partial \mathrm{x}} \\
=\frac{\partial \mathrm{u}_{0}}{\partial \mathrm{y}}+\frac{\partial \mathrm{v}_{0}}{\partial \mathrm{x}}-2 \mathrm{z}\left(\frac{\partial^{2} w_{0}}{\partial \mathrm{x} \partial \mathrm{y}}\right) \\
\gamma_{x y}=\gamma_{x y}-2 \kappa \mathrm{z}_{\mathrm{xy}}
\end{gathered}
$$


Above equation in matrix form

$$
\left\{\begin{array}{l}
\epsilon_{x} \\
\epsilon_{y} \\
\epsilon_{z}
\end{array}\right\}=\left\{\begin{array}{l}
\epsilon_{x} \\
\epsilon_{y} \\
\epsilon_{z}
\end{array}\right\}+\mathrm{z}\left\{\begin{array}{l}
\kappa_{x} \\
\kappa_{y} \\
\kappa_{z}
\end{array}\right\}
$$

Stress Resultant or in plane force and moments in terms of stresses

Let $\mathrm{N}_{x}$ and $\mathrm{N}_{y}$ are the in plane forces per unit length and $M_{x}, M_{y}$ and $M_{x y}$ be the moment per unit length.

Therefore forces in element area $=\mathrm{N}_{x}=\sigma_{x} \times 1 \times \mathrm{d}_{z}$

Force to entire area $=\int_{-h / 2}^{+h / 2} \sigma_{x} \times \mathrm{d}_{z}($ in displacement of $\mathrm{x})$

$\mathrm{N}_{x}=\int_{-h / 2}^{+h / 2} \sigma_{x} \times \mathrm{d}_{z}($ in displacement $\mathrm{x})$

$\mathrm{N}_{y}=\int_{-h / 2}^{+h / 2} \sigma_{y} \times \mathrm{d}_{z}($ in displacement of $\mathrm{y})$

$$
\mathrm{N}_{x y}=\int_{-h / 2}^{+h / 2} \tau_{x y} \times \mathrm{d}_{z}
$$

Similarly

$$
\begin{aligned}
& \mathrm{M}_{\mathrm{x}}=\int_{-h / 2}^{+h / 2}\left(\sigma_{x} \times \mathrm{d}_{z}\right) z \\
& \mathrm{M}_{\mathrm{y}}=\int_{-h / 2}^{+h / 2}\left(\sigma_{y} \times \mathrm{d}_{z}\right) z \\
& \mathrm{M}_{\mathrm{xy}}=\int_{-h / 2}^{+h / 2}\left(\tau_{x y} \times \mathrm{d}_{z}\right) z \\
& \mathrm{E}=\begin{array}{l}
\frac{\partial u}{\partial x} \\
\frac{\partial v}{\partial x}+\frac{\partial u}{\partial y}
\end{array} \quad \gamma=\left\{\begin{array}{l}
\frac{\partial w}{\partial x}+\theta x \\
\frac{\partial w}{\partial x}+\theta y
\end{array}\right\} K=\begin{array}{c}
\frac{\partial \theta x}{\partial x} \\
\frac{\partial \theta y}{\partial x}+\frac{\partial \theta x}{\partial y}
\end{array}
\end{aligned}
$$

The Force and moment for on orthotropic plate are given below:

$$
N=\left\{\begin{array}{c}
N_{x} \\
N_{y} \\
N_{x y}
\end{array}\right\} ; M=\left\{\begin{array}{c}
M_{x} \\
M_{y} \\
M_{x y}
\end{array} \quad Q=\left\{\begin{array}{l}
Q_{y} \\
Q_{x}
\end{array}\right.\right.
$$

$N=$ in plane result and forces

$M=$ Resultant moments

$Q=$ Resultant transverse shearing force

$$
\left\{\begin{array}{c}
N \\
M \\
Q
\end{array}\right\}=\left[\begin{array}{ccc}
A & B & 0 \\
B^{T} & D & 0 \\
0 & D & A_{s}
\end{array}\left\{\begin{array}{c}
E \\
K \\
\gamma
\end{array}\right\}\right.
$$

$A=$ Extensional stiffness

$B=$ Bending extensional coupling stiffnes

$D=$ Bending stiffnes

As $=$ Transverse shearing stiffnes

$$
\begin{gathered}
A=\left[\begin{array}{lll}
A_{11} & A_{12} & A_{16} \\
A_{12} & A_{22} & A_{26} \\
A_{16} & A_{26} & A_{66}
\end{array}\right] B=\left[\begin{array}{lll}
B_{11} & B_{12} & B_{16} \\
B_{12} & B_{22} & B_{26} \\
B_{16} & B_{26} & B_{66}
\end{array} ;\right. \\
D=\left[\begin{array}{lll}
D_{11} & D_{12} & D_{16} \\
D_{12} & D_{22} & D_{26} \\
D_{16} & D_{26} & D_{66}
\end{array} ; A_{s}=\left[\begin{array}{ll}
A_{55} & A_{45} \\
A_{45} & A_{44}
\end{array}\right] ;\right.
\end{gathered}
$$

\section{Moving Least SQuaRe Method}

\section{A. Moving Least Square Method}

The Gauss who is a mathematician developed a method for approximation of curves called "Method of Least Squares "in 1808 AD. The name itself indicates that the trying of the reducing or cut down the errors squares sum. Lancaster and salkauskas formulated moving least square method.

Nayroles et al 1992 used first time meshless method approximations and Bylestchko et al (1994). This idea was further extended and formulated into Element Free Gelrkin Method. In Meshless Method the assumptions are of series of monomials. These monomials are the unknowns are computed and those squared to minimize the errors at the point. At that point once the approximation is over ML's moved to another point both nodes and gauss points may be taken into consideration. The equations leads to ML's shape functions are listed below:

$$
\begin{aligned}
& \overline{\mathrm{u}}=\mathrm{a}_{1}+\mathrm{a}_{2} \mathrm{x}+\mathrm{a}_{3} \mathrm{x}^{2}+\ldots \ldots \ldots \\
& \overline{\mathrm{u}}=\sum_{i=1}^{m} \operatorname{pi}(x) \operatorname{ai}(x) \ldots \ldots . \\
& \left.\mathrm{J}=\sum_{i=1}^{n} w(x-x i) \quad{ }_{j=1}^{n} p j(x i) a j(x)-u i\right] 4 \ldots . . \\
& \mathrm{N}=\mathrm{p}^{\mathrm{t}}(\mathrm{x}) \mathrm{A}^{-1}(\mathrm{x}) \mathrm{B}(\mathrm{x}) \\
& \overline{\mathrm{u}}=m_{i=1} N i(x) u i \ldots \ldots .
\end{aligned}
$$

\section{B. Weight Functions and Polynomial Basis}

In ML's both weight functions and polynomials basis are most important they require more attention, hence these ML's methods are depends on weight function and order is to be seen. From literature we can selected those very easily. Based on weight function we may decide how much importance should give for node in numerical integration.

These criteria's should be seen before selecting the function

Here are many functions which may be used:

$$
\begin{aligned}
& \frac{2}{3}-4 r+4 r^{\wedge} 2 \text { for } \gamma \leq 1 / 2 \\
& \mathrm{~W}(\mathrm{v})=\frac{4}{3}-4 r+4 r^{\wedge} 2-\frac{4}{3} r^{\wedge} 2 \text { for } \frac{1}{2} \leq r \leq 1 \\
& 0 \text { forr }>0 \\
& \mathrm{~W}(\mathrm{r})=\left\{\begin{array}{c}
1-6 r^{2}+8 r^{3}-3 r^{4} \text { forr } \leq 1 \\
0 \text { forr }>1
\end{array}\right. \\
& \mathrm{W}(\mathrm{r})=\left\{\begin{array}{c}
1-10 r^{2}+20 r^{3}-15 r^{4}+4 r^{5} \text { forr } \leq 1 \\
0 \text { forr }>1
\end{array}\right.
\end{aligned}
$$

In the present work we considered cubic weight function. The number of nodes that must be taken into the domain, which will affect the basis of order.

$$
\begin{aligned}
& \text { Linear }=1+x+y \\
& \text { Quadratic }=1+x+y+x^{2}+y^{2}+x y \\
& \text { Cubic }=1+x+y+x^{2}+x y+y^{2}+x^{3}+x^{2} y+x y^{2}+y^{3}
\end{aligned}
$$

If mesh is regular then the influence domain size becomes very easy to handle and operate. In the present work the rectangular Meshes are used, which are suited best. 


\section{Element Free Galerkin Method}

This method is established by Nayroles et al. This is introduced for the solution of differentials equations. In this method the elements are absent. The main advantage of this method is algorithms can be easily adopted. In case of cracking problems due to the absent of elements, the true crack may be influenced. Where cracks can't be cut through element in the EFGM the cracks can be crosses in between 2 nodes. EFGM have larger convergence rate then the finite element method and completely depends on the weight functions.

\section{Numerical Integration}

In EFGM numerical integration is used to divide the entire domain in to the simple cells. These are used to define the quadrature points were the coefficient of stiffness may calculate. The support domain of the point is defined by considering gauss integration points. The support domain is defined for a point but the influence domain is defined only for a node

In this work, Meshless shape function is used \& the nodal stiffness matrix is obtained.

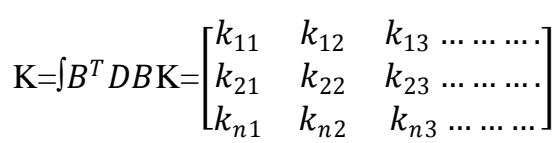

Lagrange multiplier worked on the principle which the gradient of the main functions and the gradient constraint function is same at optimum point i.e. common for both the functions. The ML's shape functions are used to find the values of Lagrange multiply at nodes Lagrange polynomials can be used.

To find the parameters like dispatch, furies in the domain and at the boundary of the plate's gauss points and the integration cells are used. Generally $4 \times 4$ gauss quadrature is used in this present work. Further $6 \times 6$ Gauss and $8 \times 8$ gauss quadrature are also considered.

\section{RESULTS AND DISCUSSIONS}

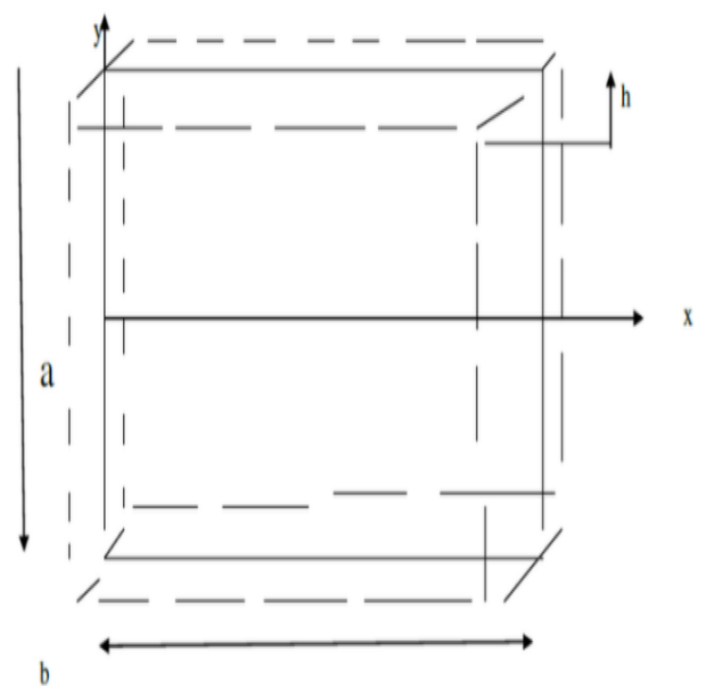

Figure 4: Section of Plate
A. Output Results For this Study:Transeverse Displacement Actual Displacement

\begin{tabular}{|l|l|l|l|}
\hline RKPM & FEM & WANG[1] & J.NREDDY[13] \\
\hline 1.914 & 1.50 & 1.941 & 1.9468 \\
\hline
\end{tabular}

Parametric Study

\begin{tabular}{|l|l|l|l|l|}
\hline Integration Scheme & Ratio ah & Particle Distribution & Particle Distribution & Particle Distribution \\
\hline & & $11 \times 11$ & $15 \times 15$ & $17 \times 17$ \\
\hline $2 \times 2$ & 10 & 2.1615 & 2.1901 & 2.2344 \\
\hline $2 \times 2$ & 20 & 1.6343 & 1.7808 & 1.8201 \\
\hline
\end{tabular}

Example 2: A 4 Layered symmetric Cross ply $\left[0^{\circ} / 90^{\circ} / 90^{\circ} / 0^{\circ}\right]$ orthotropic plates considered in J N Reddy [ 1]. The plate simply supported all along the sides of plates carrying uniformly distributed load, \& the properties as given below.

$$
\begin{gathered}
\mathrm{E}_{1}=25 ; \mathrm{E}_{2}=1 ; \\
\mathrm{G}_{12}=\mathrm{G}_{13}=0.5 \mathrm{E}_{2} ; \\
\mathrm{G}_{23}=0.2 \mathrm{E}_{2} ; \\
\gamma_{12}=\gamma_{23}=\gamma_{13}=0.25
\end{gathered}
$$

\begin{tabular}{|c|l|c|c|}
\hline$a / h$ & Particle Size & $\bar{w}$ & $\bar{w}[13]$ \\
\hline \multirow{3}{*}{10} & $11 \times 11$ & $1.0517[-0.0298]$ & \\
\cline { 2 - 3 } & $17 \times 17$ & $1.0719[-0.05]$ & \\
\cline { 2 - 3 } & $21 \times 21$ & $1.0789[-0.057]$ & 1.0219 \\
\hline \multirow{3}{*}{20} & $11 \times 11$ & $0.6779[0.0793]$ & \\
\cline { 2 - 3 } & $17 \times 17$ & $0.7159[0.0413]$ & \multirow{4}{*}{0.7572} \\
\cline { 2 - 3 } & $21 \times 21$ & $0.7250[0.0322]$ & \\
\hline
\end{tabular}

\section{CONCLUSION}

In the present study orthotropic laminated composite plates are used. The analysis has been done by Element Free Gelerkin Method (EFGM) and Shape functions are obtained from Meshless Method of EFGM. Even Though analysis may be done with FEM, this study may be extended further study of fracture mechanics of laminated composite plates. FSDT theory has been applied moderately to thick to thin plates efficiently. The weigh function, integration schemes, nodal density, and proper algorithm for the performance of Meshless Method. Conclusions summary from present work:

- MLS shape function provides considerably accurate results for all the problems of plate and laminates for bending.

- The cubic weight function that was considered influenced the test function which affected the end results.

- Higher the order, minimum number of nodes were required for decent approximation.

- Through validation, it was clear that the errors for transverse displacement were comparatively less. Therefore the Meshless Method can be efficiently applied to Composite Laminated Plates. 


\section{FUTURE SCOPE}

1) The applications of Meshless Method using higher order plate theories.

2) Vibration and Buckling analyses may be conduct on laminates using FSDT of Meshless Method as an extension of the current study.

3) Analysis of Transverse stresses can be extended.

4) Meshless Method can be extended to curved shells.

5) Problems of different boundary conditions can be applied.

\section{REFERENCES}

[1]. J. Wang, K.M. Liew, M.J. Tan, S. Rajendran, "Analysis of rectangular laminated composite plates via FSDT meshless method", International Journal of Mechanical Science, Pp. 1276-1293, 2002.

[2]. J.N. Reddy, "On refined computational models of composite laminates", International Journal for numerical Methods in Engineering, Vol. 27, Pp. 361-382, 1989.

[3]. B. Nayroles, G. Touzot, and P. Villon, "Generalizing the finite element method: Diffuse approximation and Diffuse elements", Computational Mechanics., Vol. 10, Pp. 305-318, 1992.

[4]. T. Belytschko et al, "Element-free galerkin methods", International Journal for Numerical Methods in Engineering Vol. 37, Pp. 229-256, 1994.

[5]. I. Kaljevi and S. Saigal "An improved element free galerkin formulation", International Journal for Numerical Methods in Engineering, Vol. 40, Pp. 2953-2974, 1997.

[6]. Y.X. Mukherjee and S. Mukherjee, "Boundary node method for potential problems", International Journal for Numerical Methods in Engineering Vol. 40, Pp. 797-815, 1997.

[7]. M.K. Singha, and D. Rupesh, "Nonlinear vibration of symmetrically laminated composite skew plates by finite element method", International Journal of Non-Linear Mechanics Vol. 42, Pp. 1144-1152, 2007.

[8]. B. Sidda Reddy et al., "Bending analysis of laminated composite plates using finite element method", International Journal of Engineering, Science and Technology, Vol. 4, No. 2, Pp. 177-190, 2012. 